\title{
Patterns of postmating reproductive isolation in a newly discovered species pair, Aquarius remigis and $A$. remigoides (Hemiptera; Gerridae)
}

\author{
SHARON L. GALLANT \& DAPHNE J. FAIRBAIRN* \\ Department of Biology, Concordia University, 1455 de Maisonneuve Blvd., West, Montreal, Quebec, Canada, \\ H3G $1 M 8$
}

\begin{abstract}
Aquarius remigoides Gallant and Fairbairn has recently been described as specifically distinct from $A$. remigis (Say) based upon genetic and morphological data. Both species are common, semiaquatic bugs (Hemiptera; Gerridae) found on the surface of streams and small rivers. Allozyme studies have shown them to be more distantly related than most other congeneric species in the Gerridae, with significant barriers to gene flow where their ranges abut. We assess postmating reproductive isolation between $A$. remigoides and $A$. remigis, using a bracket cross design with five sampling sites along a north-south cline traversing the hybrid zone. We also report the results of long-distance conspecific crosses of $A$. remigis, using populations from California and Quebec. Neither these nor the intraspecific bracket crosses reveal any evidence of isolation by distance within species. However, heterospecific crosses show significantly reduced fertility, hatching success, survival of both sexes to eclosion (final moult) and percentage of males. Egg production is not influenced by cross type, and we found no evidence of hybrid sterility in either sex. Analyses of genotypic frequencies at three isozyme loci in eight hybrid and 22 pure populations reveal significant deficiencies of heterozygotes in hybrid populations. The proportion of males is also significantly lower in hybrid populations than in pure populations. The laboratory and field results indicate that postmating isolation between these two species occurs in the form of severe reductions in both the fertility of heterospecific crosses and the viability of hybrids, particularly hybrid males. Genetic mechanisms for Haldane's rule and asymmetries in the reciprocal heterospecific crosses are discussed.
\end{abstract}

Keywords: bracket crossing, gene flow, Haldane's rule, heterozygote deficiency, hybrid zone, waterstrider.

\section{Introduction}

Interspecific hybridization is common in many plant and animal taxa, and hybrid zones provide a valuable opportunity to study the dynamics of reproductive isolating barriers between species (Harrison, 1990, 1993). The barriers to gene flow are expressed most commonly as one or a combination of reduced fertility of heterospecific crosses, hybrid inviability or hybrid sterility, with the last two effects expressed primarily in the heterogametic sex (Haldane, 1922; Wu \& Davis, 1993).

Field studies of hybrid zones based on molecular or chromosomal markers typically find a deficiency

\footnotetext{
*Correspondence. E-mail: fairbrn@vax2.concordia.ca
}

of hybrid individuals, indicative of restricted gene flow between species (Harrison, 1993). Laboratory crosses between such species allow researchers to assess the roles of pre- and postzygotic reproductive isolation, and to investigate patterns of speciation (e.g. Virdee \& Hewitt, 1994). In this paper, this approach was used to characterize reproductive isolation between a common, stream-dwelling waterstrider, Aquarius remigis (Say) (Hemiptera: Gerridae), and its newly described congener, $A$. remigoides Gallant and Fairbairn.

Aquarius remigis is found on the surfaces of streams and small rivers from the Canadian Yukon and North-West Territories south to Mexico and Guatemala (Drake \& Harris, 1934). Populations from the south-eastern U.S.A., formerly considered 
to be $A$. remigis, are now classified as $A$. remigoides, and a hybrid zone between these species runs eastwest through Pennsylvania and New Jersey (Fig. 1a; Gallant et al., 1993; Gallant \& Fairbairn, 1996).
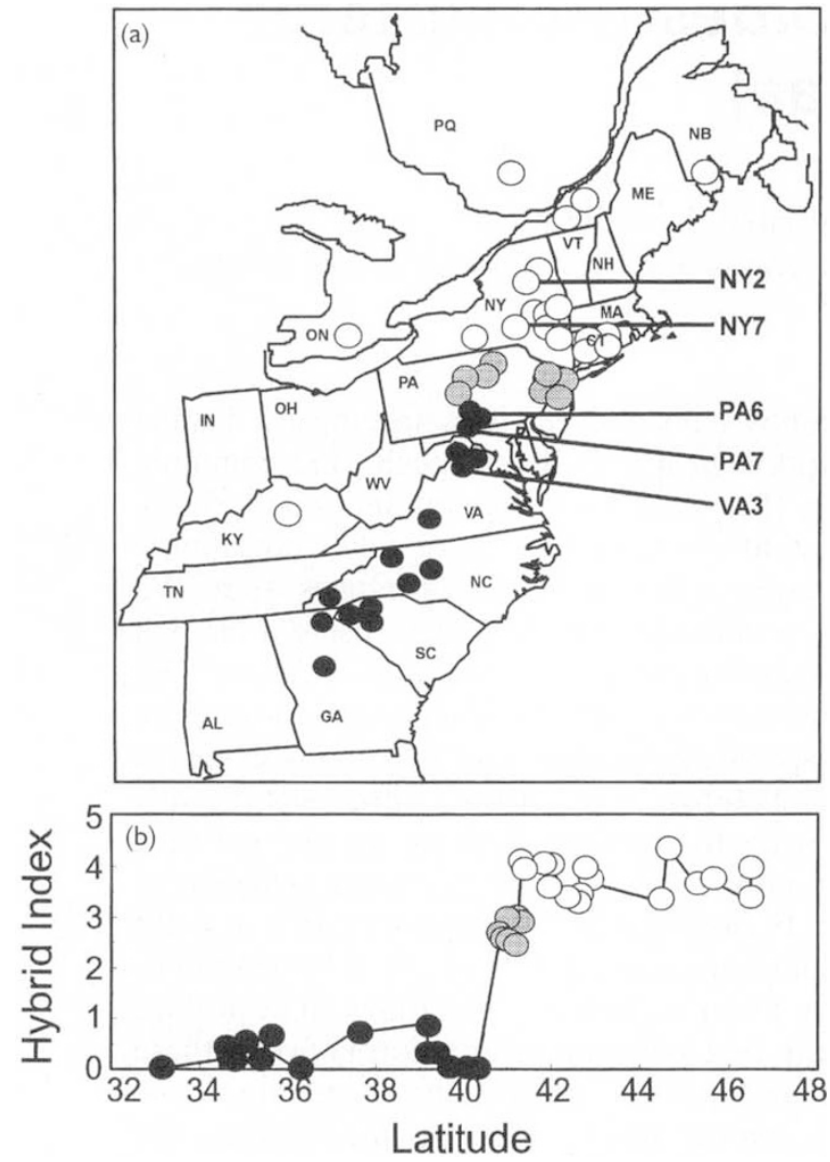

Fig. 1 (a) Locations of all eastern populations that have been genetically characterized, based on hybrid index scores, as pure populations of Aquarius remigis (open circles), pure populations of $A$. remigoides (closed circles) or hybrid populations (grey circles). The sampling sites used for the bracket crosses are indicated: Johnsburg, NY (NY2), and Grafton, NY (NY7), for A. remigis; Hoernerstown, PA (PA6), Webster Mills, PA (PA7), and East Winchester, VA (VA3), for $A$. remigoides. (b) Hybrid index as a function of latitude for populations along the longitudinal Appalachian Mountain transect (i.e. excluding the populations from Kentucky and Ontario). The hybrid index is the sum of the frequencies of $A$. remigisspecific alleles across the five loci that differentiate $A$. remigis from $A$. remigoides (Gallant et al., 1993; Gallant \& Fairbairn, 1996): phosphogluconate dehydrogenase (PGD-1, EC 1.1.1.44), superoxide dismutase (SOD-1, EC 1.15.1.1), malate dehydrogenase ( $M D H-1, \mathrm{EC} 1.1 .1 .37)$, glycerol-3-phosphate dehydrogenase (GPD-1, EC 1.1.1.8), and alkaline phosphatase (ALP-1, EC 3.1.3.1). The index can vary from five, for a population fixed for the $A$. remigis-specific alleles, to zero for a population fixed for the $A$. remigoides-specific alleles.
Cluster analysis of Rogers's genetic distances (based on 13 allozyme loci) and comparisons of the male genitalia have allowed characterization of 63 populations as $A$. remigis or $A$. remigoides (Preziosi \& Fairbairn, 1992; Gallant et al., 1993; Gallant \& Fairbairn, 1996). A hybrid index (Brennan \& Fairbairn, 1995), based upon the five allozyme loci that allow differential diagnosis of the two species, graphically illustrates their genetic distinctiveness, as well as the geographical abruptness of the hybrid zone (Fig. 1b).

In spite of their genetic and morphological distinctiveness, the two species seem to be ecologically similar. Both are large $(11-17 \mathrm{~mm}$ in total length), active scavengers and predators, feeding mainly on arthropods trapped in the surface film. Adults overwinter in reproductive diapause, and both sexes mate many times over a prolonged reproductive period in the spring and early summer. Aquarius remigis has only one generation per year throughout most of Canada and the northern U.S.A., but becomes bivoltine in warmer regions (Blanckenhorn \& Fairbairn, 1995). The phenology of $A$. remigoides is not known, but our field sampling experience suggests similar transitions from univoltinism to bivoltinism, mediated by both latitude and altitude.

This paper examines whether genetic divergence between this newly identified species pair has proceeded sufficiently to produce reproductive isolation. We combine a laboratory crossing experiment designed to detect postmating reproductive isolation with a field survey of genotype frequencies and sex ratios, designed to detect deficiencies of heterozygotes and/or of males in hybrid populations. The laboratory crossing protocol is a 'bracket crossing' design (Virdee \& Hewitt, 1994) with five sampling sites along a north-south cline traversing the hybrid zone (Fig. 1). We compare the resulting intrasite and intersite conspecific and heterospecific crosses with respect to the number and fertility of eggs laid by parental females, and the viability, fertility and proportion male in the $F_{1}$ generation.

\section{Materials and methods}

\section{Laboratory crosses}

We collected prereproductive adult $A$. remigis and $A$. remigoides from five natural populations (Fig. 1) in September 1994 and overwintered them in a laboratory refrigerator from 26 September until 1 March, when we set up the experimental crosses. Parental crosses consisted of all possible intrasite 
and intersite crosses, with the following omissions dictated by sample size: female $A$. remigoides (VA3) $\times$ male $A$. remigoides $(\mathrm{PA} 7$ ), and all crosses involving $A$. remigoides (PA6) except those with $A$. remigis from NY2. For each of the 17 parental crosses, we put 10 females and 10 males in a $40 \times 50 \mathrm{~cm}$ tank containing water to a depth of approximately $7 \mathrm{~cm}$, with food (frozen Drosophila and crickets), oviposition and resting sites (floating pieces of foam rubber) and air bubblers to provide current. Tanks were kept at room temperature (mean $=20.9^{\circ} \mathrm{C}, \mathrm{SD}=2.1^{\circ} \mathrm{C}$ ) under an $18 \mathrm{~h}$ light: $6 \mathrm{~h}$ dark photoperiod.

After 5 days, we removed and discarded all of the oviposition sites. Thereafter, we collected all eggs from each tank every 3 days for 4 weeks. On each collecting day, we counted all eggs and then seeded each $25 \times 40 \mathrm{~cm} F_{1}$-rearing tank with 50 eggs for a total of 450 eggs per cross. Rearing tanks were equipped as above and kept at $21^{\circ} \mathrm{C}$ with a photoperiod of $12 \mathrm{~h}$ light:12 $\mathrm{h}$ dark. At the third larval instar, sex could be unambiguously determined and nymphs were separated into tanks containing solely males or females for each cross.

Nineteen days after seeding each rearing tank, we examined the oviposition sites under a dissecting microscope to determine egg fertility and hatching success. Eggs that had failed to hatch were scored as infertile (a uniformly off-white egg with no eyespots or internal structure visible), or post-eyespot failure (eyespots normally appear within the first 2 or 3 days of development).

In order to assess the fertility of $\mathrm{F}_{1}$ hybrids, we paired each hybrid male with $\mathrm{F}_{1}$ females of three types: $A$. remigis, $A$. remigoides and hybrid. Pairings were consecutive, with each pair remaining together for 7 days. The order of female types was different for each male. This design maximized the number of $F_{1}$ crosses possible with the limited number of hybrid males surviving to adulthood $(N=5)$. Hybrid females were more common, and so each female was paired for 7 days with a single pure $F_{1}$ male.

In order to assess the maximum expected isolation by distance, we crossed $A$. remigis collected as prereproductive adults from south-western Quebec (PQ) and the Solano and Napa counties of northern California (CA) (number of pairs $=5 \quad \mathrm{PQ} \times \mathrm{PQ}, 4$ $\mathrm{CA} \times \mathrm{CA}, 6 \mathrm{CA}$ females $\times \mathrm{PQ}$ males, $6 \mathrm{PQ}$ females $\times$ CA males). The protocol was as above, except that rearing was at room temperature and $16 \mathrm{~h}$ light: $8 \mathrm{~h}$ dark, and the crosses were compared only with respect to egg fertility, assessed at 16 days after oviposition.

Where parametric statistical methods are presented, the results have been confirmed with nonparametric equivalents. Paired comparisons following ANOVA are based on multiple $t$-tests, with Bonferroni corrections for multiple comparisons (Rice, 1989).

\section{Allozyme methods}

Locations of sampling sites for electrophoretic analyses are shown in Fig. 1. Sample sizes ranged from 8 to $216($ mean $=60 ; S D=42)$. All individuals were captured as adults and stored separately in 0.5 $\mathrm{mL}$ Eppendorf vials at $-40^{\circ} \mathrm{C}$. We used horizontal starch gel electrophoresis to detect 13 loci (cf. Preziosi \& Fairbairn 1992; Gallant et al. 1993). Tests of goodness-of-fit to Hardy-Weinberg equilibria are based on genotypic frequencies at the three loci that were polymorphic within hybrid populations: phosphogluconate dehydrogenase (PGD-1), malate dehydrogenase $(M D H-1)$ and superoxide dismutase (SOD-1).

\section{Results}

\section{Laboratory crosses}

The number of eggs laid per cross did not differ significantly among parental cross types (Fig. 2a; $F_{5,11}=1.062, P=0.431$ ). However, the proportion of fertile eggs did differ $\left(F_{5,11}=9.085, P=0.001\right)$, being significantly reduced in the heterospecific crosses (Fig. 2b). The reciprocal heterospecific crosses also differed significantly from each other, with lower fertility in crosses with $A$. remigoides mothers (Fig. 2b). The proportion of fertile eggs that hatched successfully showed the same pattern (Fig. 2c; $\left.F_{5,11}=7.345, P=0.003\right)$. Overall, the percentage of eggs from heterospecific crosses that failed to hatch because of infertility or developmental failure was 61 per cent for crosses with $A$. remigis mothers and 82 per cent for crosses with $A$. remigoides mothers. By comparison, only 21 per cent of eggs from conspecific crosses failed to hatch. The number of offspring reaching adulthood showed the same pattern, although the difference between the heterospecific crosses is not statistically significant (Fig. 2d; $\left.F_{1,15}=541.085, \quad P<0.0001\right)$. Conspecific crosses produced an average of 31 adults per cross, whereas heterospecific crosses produced an average of only two adults. The sex ratio among adult hybrids was significantly female-biased (15 females to five males; $\left.\chi_{1}^{2}=5, P<0.05\right)$. In contrast, conspecific crosses produced balanced sex ratios: 50.0 per cent male for $A$. remigis and 49.5 per cent male for $A$. remigoides. 


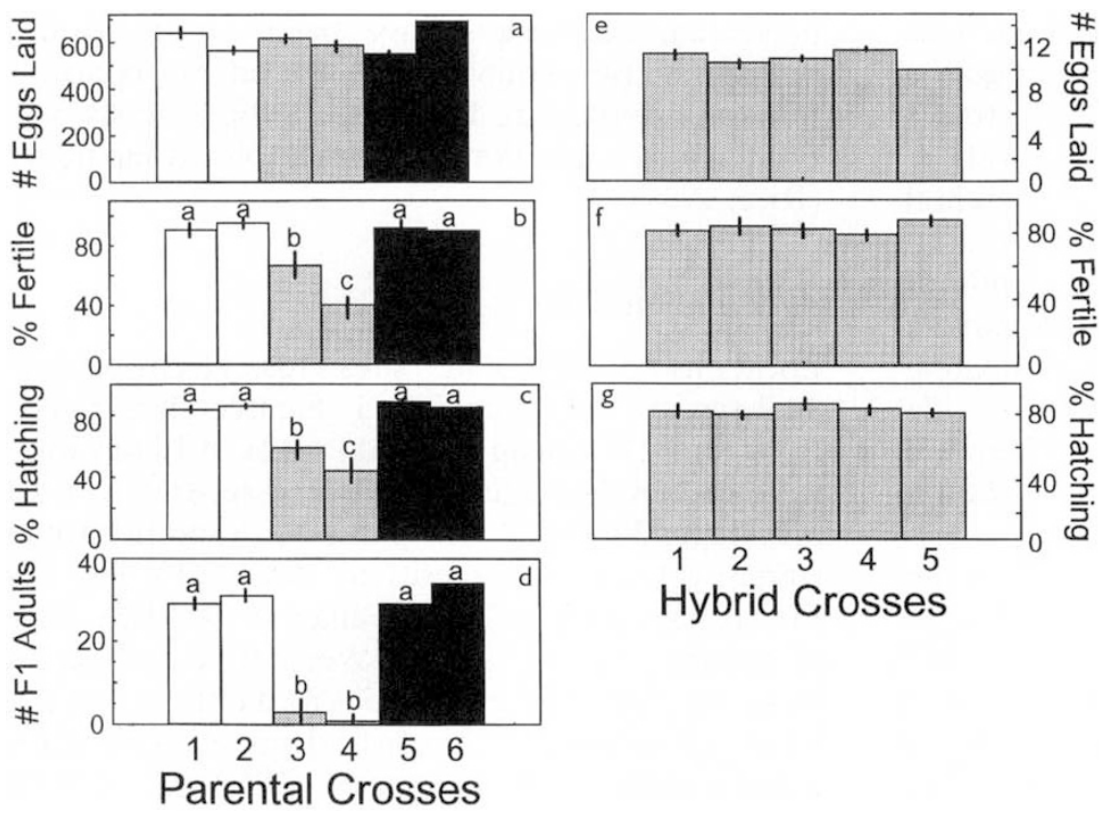

Fig. 2 Results of conspecific Aquarius remigis (white bars), heterospecific (grey bars) and conspecific $A$. remigoides (black bars) crosses. (a-d) Parental crosses: $1, A$. remigis, intrasite; $2, A$. remigis, intersite; 3 , $A$. remigis females $\times A$. remigoides males; $4, A$. remigoides females $\times A$. remigis males; $5, A$. remigoides, intrasite; $6, A$. remigoides, intersite. (e-g) Crosses involving $\mathrm{F}_{1}$ hybrids: 1 , hybrid $\times$ hybrid; $2, A$. remigis females $\times$ hybrid males; $3, A$. remigoides females $\times$ hybrid males; 4 , hybrid females $\times A$. remigis males; 5 , hybrid females $\times A$. remigoides males. All bars indicate mean values per cross type. Vertical bars are $\pm 1 \mathrm{SD}$. (a and e) Total number of eggs laid; (b and $f$ ) percentage of fertile eggs; (c and $\mathrm{g}$ ) percentage of fertile eggs hatching; and (d) total number of $F_{1}$ adults. Significant differences are indicated by different letters above the bars. Note that the number of eggs laid is based on 10 females over 4 weeks for parental crosses, but only one female over one week for hybrid crosses.
The crosses of populations from Quebec and California revealed no evidence of isolation by distance in $A$. remigis: the proportion of fertile eggs did not differ significantly among the four cross types $\left(F_{3,17}=1.083, P=0.385\right)$, and the average fertility was actually slightly higher for the inter-regional crosses (55 per cent) than for the intra-regional crosses (44 per cent).

Hybrid $\left(\mathrm{F}_{1}\right)$ cross types did not differ significantly with respect to the number of eggs laid (Fig. 2e; $F_{4,15}=1.906, \quad P=0.162$ ), fertility (Fig. 2f; $F_{4,15}=0.932, P=0.472$ ) or hatching success (Fig. 2g; $\left.F_{4,15}=0.056, P=0.994\right)$.

\section{Allozyme results}

Of the eight hybrid populations (Fig. 1), six showed significant heterozygote deficiencies (Fig. 3). Among populations outside the hybrid zone, 22 were polymorphic for $P G D-1, S O D-1$ or $M D H-1$, but only two of these showed significant heterozygote deficiency (Fig. 3). We used meta-analysis (Mullen \& Rosenthal, 1985) to quantify this difference between hybrid and nonhybrid populations. Formally, each meta-analysis estimates the probability of obtaining the observed distribution of probability values if heterozygotes are not less common than expected (i.e. $\mathrm{H}_{0}$ : Selander's $D \geq 0 ; \mathrm{H}_{\mathrm{A}}$ : Selander's $D<0$; Fig. 3). At the $P G D-1$ locus, these probabilities are
0.331 for $A$. remigis, 0.185 for $A$. remigoides and $10^{-7}$ for hybrid populations. Thus, $P G D-1$, our most polymorphic locus, clearly indicates heterozygote deficiencies in hybrid populations. SOD-1 and $M D H-1$ are not polymorphic in sufficient numbers of populations for separate meta-analyses. However, as no population was polymorphic at both loci, we combined them to yield a total sample of 17 independent assays of heterozygote deficiency. Metaanalysis of the combined data yielded probabilities of 0.022 for $A$. remigis, 0.391 for $A$. remigoides and $10^{-4}$ for hybrid populations. As for $P G D-1$, these data indicate highly significant heterozygote deficiencies in hybrid populations. The slight deficiency of heterozygotes suggested for $A$. remigis is entirely caused by deficiencies at $S O D-1 ; M D H-1$ shows no corresponding trend (Fig. 3). The deficiencies of $S O D-1$ heterozygotes may represent scoring errors, as the two most extreme populations (NY1 and NY3) were among the first populations analysed (Gallant et al., 1993), and heterozygotes at this locus tend to be difficult to score (they are faint and can be missed, leading to a possible excess of 'no score' values for heterozygous individuals). However, we refrain from further speculation because the significance of the overall probability is doubtful: we performed six separate meta-analyses, and our critical probability level for the $A$. remigis analysis (using a sequential Bonferroni correction; Rice 1989) 

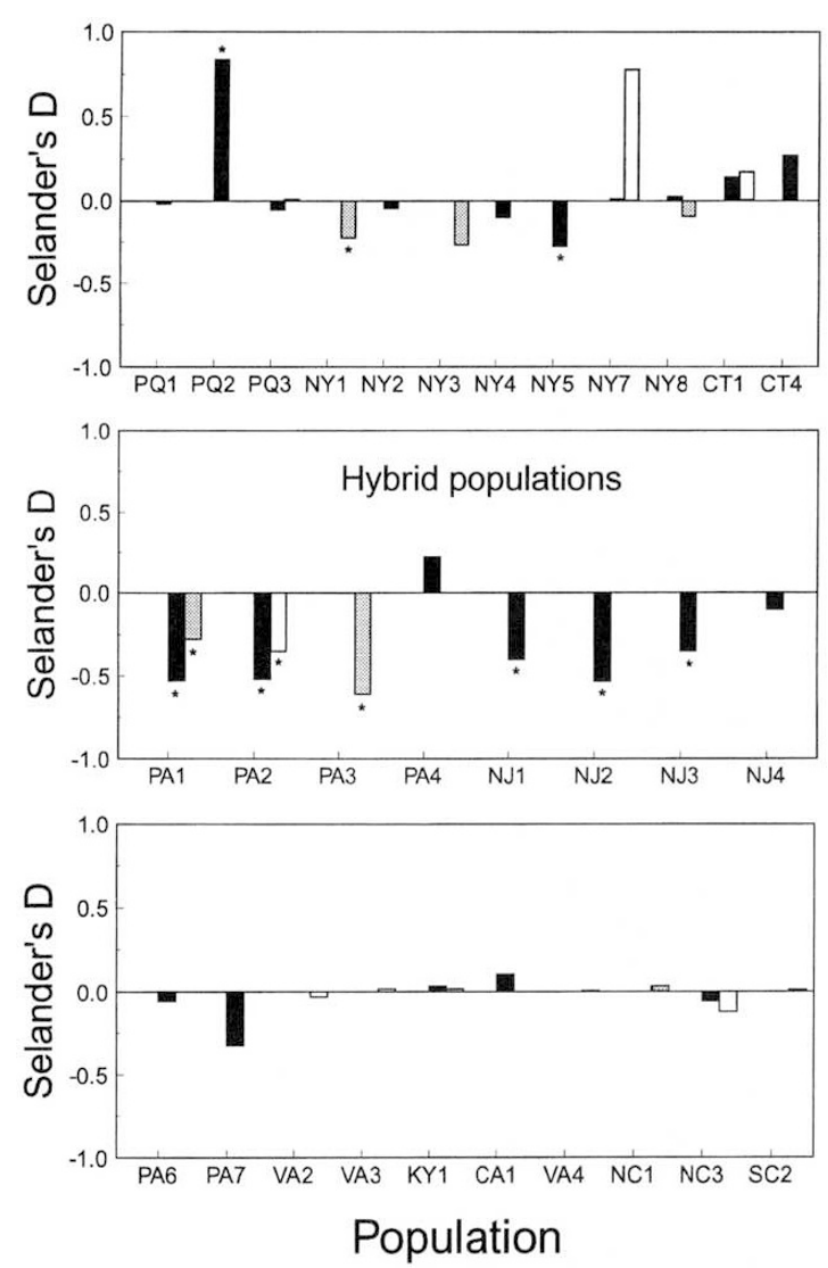

Fig. 3 Selander's index of heterozygote deficiency or excess $\left[D=\left(H_{\text {obs }}-H_{\text {exp }}\right) / H_{\text {exp }}\right.$; Selander, 1970) for the three loci polymorphic in populations of Aquarius remigis and $A$. remigoides and in hybrid populations: $P G D-1$ (black bars), SOD-1 (grey bars) and $M D H-1$ (white bars). The 30 populations that were polymorphic at one or more of these loci are ordered from most northern (left side of upper panel) to most southern (right side of lower panel). The middle panel shows hybrid populations. Asterisks denote statistically significant deviations from HardyWeinberg equilibrium expectations, with probabilities not corrected for multiple comparisons.

should therefore be $0.05 / 4=0.0125$, rendering the apparent heterozygote deficiency nonsignificant. Our main conclusion is that, for all of the polymorphic loci, hybrid populations consistently show highly significant heterozygote deficiencies, and such deficiencies are rare in pure populations of either species.

\section{Proportion of males in field populations}

We tested the hypothesis of male deficiency within hybrid populations by comparing the mean propor- tion of adult males in eight hybrid (mean $=0.21$, $\mathrm{SD}=0.18$ ) and 18 nonhybrid (mean $=0.48$, $\mathrm{SD}=0.06$ ) populations for which we have data on sex ratio. Hybrid populations contained a significantly lower proportion of males than nonhybrid populations $\left(t_{7.8}=4.173, \quad P<0.003\right.$; corrected for inequality of variances).

\section{Discussion}

Interspecific hybridization is common among insect species and has been described previously in the Gerromorpha (e.g. Spence, 1990; Zimmermann \& Scholl, 1993). Thus, the ability of Aquarius remigis and $A$. remigoides to hybridize is not surprising. However, our laboratory and field studies indicate that gene flow between these species is severely restricted by postmating isolating barriers. As a result of both reduced fertility and reduced offspring viability, heterospecific crosses produce only about 6 per cent as many $F_{1}$ adults as conspecific crosses. Further, the sex ratio in hybrid adults is strongly female-biased, indicating a disproportionate loss of hybrid males. Significant deficiencies of both heterozygotes and males in hybrid field populations indicate that similar postmating barriers operate in natural populations. In the laboratory, the hybrids that do survive are fully fertile and produce viable offspring both when mated with each other and when backcrossed to the parental types. Thus, the barriers to gene flow appear to occur only in the initial crosses between $A$. remigis and $A$. remigoides.

Laboratory crosses between another congeneric pair of waterstrider species, Limnoporus dissortis and L. notabilis, provide an interesting comparison. In contrast to our results for Aquarius, Spence (1990) found that these crosses were fully fertile. Significant hybrid breakdown was expressed as reduced hatching success of $F_{1}$ hybrids and of eggs from backcrosses of hybrid males to females of their maternal species. The reduction in $F_{1}$ hatching success was of similar magnitude to that observed in this study (40-60 per cent for heterospecific, intersite crosses) and, as in this study, was asymmetric for reciprocal crosses. The two studies also agree in finding no evidence of isolation by distance within species. These comparisons indicate that the barriers to gene flow between $A$. remigis and $A$. remigoides operate earlier and are initially more complete than those between $L$. dissortis and $L$. notabilis, reducing egg fertility as well as $F_{1}$ viability. However, the Limnoporus system includes partial barriers to gene flow through backcrossing that are not found in our Aquarius system. 
We found significant deficiencies of males in hybrid field populations and among the offspring of heterospecific laboratory crosses. In contrast to this, Spence (1990) found a significant deficiency of females in his crosses between Limnoporus dissortis and $L$. notabilis. Our results are more typical. Reduced viability and/or sterility in the heterogametic sex, in this case males, is found so frequently in studies of hybridization that it has achieved the status of an ecological rule: 'Haldane's rule' (Haldane, 1922). Current theory suggests that the sterility and inviability aspects of Haldane's rule are separate evolutionary phenomena and are unlikely to have a common genetic basis (Wu \& Davis, 1993). Our finding of inviability only is therefore not surprising. The simplest hypothesis for hybrid inviability is disruptive epistasis between the male's single $\mathrm{X}$ chromosome and one set of foreign autosomes. The $F_{1}$ females possess an $X$ chromosome from both parental species, and hence avoid these detrimental interactions (Turelli \& Orr, 1995).

Crosses between $A$. remigoides females and $A$. remigis males had significantly reduced fertility and hatching success when compared with the reciprocal crosses. Asymmetries between reciprocal heterospecific crosses are common (Harrison, 1990) and have previously been found in crosses between Aquarius cinereus and $A$. najas (Zimmermann \& Scholl, 1993), and between Limnoporus dissortis and $L$. notabilis (Spence, 1990). In both of these examples, the reciprocal crosses differ in mating frequency, with significantly reduced mating frequency between males of the larger species and females of the smaller species. We found no evidence of asymmetries in mating frequency between reciprocal crosses (S. L. Gallant, unpublished data), perhaps because $A$. remigoides and $A$. remigis differ less in size than the other species pairs (Brennan \& Fairbairn, 1995; Gallant \& Fairbairn, 1996). Thus, our asymmetry was entirely as a result of postmating reproductive barriers. Zimmermann \& Scholl (1993) did not analyse postmating barriers, but Spence (1990) provides clear evidence of differences in hatching success between reciprocal crosses, indicating that postmating barriers are an important component of the asymmetry of Limnoporus dissortis $\times L$. notabilis crosses. Recent research suggests that asymmetry in postmating barriers between species may reflect genomic imprinting (Varmuza, 1993). This process, defined as 'an epigenetic, gamete-of-origin dependent modification of the genome' (Monk, 1988), may cause the genomes of males and females of hybridizing species to be functionally nonequivalent, leading to asymme- tries in the fitness of reciprocal crosses. Genomic imprinting may, therefore, provide a general, functional explanation for the commonly observed asymmetries in heterospecific crosses.

We have previously proposed that the genetic divergence between $A$. remigis and $A$. remigoides is the result of isolation during the Pleistocene followed by secondary contact (Gallant et al., 1993). Evidence in support of this hypothesis as opposed to the alternative hypothesis of primary intergradation (differentiation in situ) includes a lack of clinal variation in allozyme frequencies within species, an absence of disjunction in habitat and climatic variables across the hybrid zone, covariance of morphometric and allozyme differentiation (Brennan \& Fairbairn, 1996) and the phylogenetic relationships among populations from across North America, as assessed by allozyme frequencies. Our laboratory crosses provide further support for the hypothesis of secondary intergradation by revealing a complete absence of isolation by distance within either species. Aquarius remigis occurs across North America in genetically isolated populations (Preziosi \& Fairbairn, 1992), yet reproductive isolation has not evolved even between populations separated by a continent (California and Quebec). These results and the results of our bracket crosses provide strong evidence that genetic isolation across the hybrid zone is much greater than would be expected based on the current geographical separation of these populations. Past isolation of populations in Pleistocene refugia east and west of the Appalachian crest, with subsequent northward recolonization and secondary contact remains the more viable hypothesis.

The results of our bracket crosses together with our allozyme data have allowed us to confirm the specifically distinct status of $A$. remigoides, describe the postmating reproductive barriers between $A$. remigis and $A$. remigoides and clearly delineate the northern portion of the hybrid zone between these species. Interspecific crosses are characterized by greatly reduced fertility and $F_{1}$ viability, but we found no detectable reduction in the reproductive success of surviving hybrids. Given the rarity of male hybrid adults, we suggest that limited gene flow between these species in natural populations probably occurs primarily through backcrossing of female hybrids to parental types. Further studies of field populations will be required to test this hypothesis, as well as to ascertain the importance, if any, of premating barriers, such as differential mating frequencies or habitat use. 


\section{Acknowledgements}

We thank D. A. Roff for assistance with sample collection, A. Sih for providing the sample from Kentucky and S. Alexander for technical assistance. This research was supported by a grant to D. J. F. from the Natural Sciences and Engineering Research Council of Canada.

\section{References}

BLANCKENHORN, w. U. AND FAIRBAIRN, D. J. 1995. Life history adaptation along a latitudinal cline in water striders, Aquarius remigis (Heteroptera: Gerridae). $J$. Evol. Biol., 8, 21-41.

BRENNAN, J. M. AND FAIRBAIRN, D. J. 1995. Clinal variation in morphology among eastern populations of the waterstrider, Aquarius remigis Say (Hemiptera: Gerridae). Biol. J. Linn. Soc., 54, 151-171.

DRAKE, C. J. AND HARRIS, H. M. 1934. The Gerrinae of the Western Hemisphere (Hemiptera). Ann. Carneg. Mus., 23, 179-241.

Gallant, S. L. AND Fairbairn, D. J. 1996. A new species of Aquarius from the southeastern U. S. A., with electrophoretic analysis of the clade containing Gerris, Limniporus, and Aquarius (Hemiptera: Gerridae). Ann. Entomol. Soc. Am., 89, 637-644.

GAllaNT, S. L., PREZIOSI, R. F. AND FairbaiRn, D. J. 1993. Clinal variation in eastern populations of the waterstrider Aquarius remigis: gradual intergradation or discontinuity? Evolution, 47, 957-964.

HALDANE, J. B. S. 1922. Sex ratio and unisexual sterility in hybrid animals. J. Genet., 12, 101-109.

HARRISON, R. G. 1990. Hybrid zones: windows on the evolutionary process. Oxford Surv. Evol. Biol., 7, 69-128.

HARRISON, R. G. 1993. Hybrid Zones and the Evolutionary Process. Oxford University Press, New York.

MONK, м. 1988. Genomic imprinting. Genes Dev, 2, 921-925.

MULLEN, B. AND ROSENTHAL, R. 1985. BASIC Meta-Analysis: Procedures and Programs. Lawrence Erlbaum Assoc., Hillsdale, NJ.

PREZIOSI, R. F. AND FAIRBAIRN, D. J. 1992. Genetic population structure and levels of gene flow in the stream dwelling waterstrider, Aquarius $(=$ Gerris $)$ remigis (Hemiptera: Gerridae). Evolution, 46, 430-444.

RICE, w. R. 1989. Analyzing tables of statistical tests. Evolution, 43, 223-225.

SELANDER, R. K. 1970. Behavior and genetic variation in natural populations. Am. Zool., 10, 53-66.

SPENCE, J. R. 1990. Introgressive hybridization in Heteroptera: the example of Limnoporus Stål (Gerridae) species in western Canada. Can. J. Zool., 68, 1770-1782.

TURELLI, M. AND ORR, H. A. 1995. The dominance theory of Haldane's rule. Genetics, 140, 389-402.

VARMUZA, s. 1993. Gametic imprinting as a speciation mechanism in mammals. J. Theor. Biol., 164, 1-13.

ViRDEE, S. R. AND HEwITT, G. M. 1994. Clines for hybrid dysfunction in a grasshopper hybrid zone. Evolution, 48, 392-407.

wU, C. AND DAvis, A. w. 1993. Evolution of postmating reproductive isolation: the composite nature of Haldane's rule and its genetic bases. Am. Nat., 142, 187-212.

ZIMMERMANN, M. AND SCHOLL, A. 1993. Specific status of Aquarius cinereus (Puton) and $A$. najas (DeGeer) (Hemiptera: Gerridae) and the extent of hybridization in the Mediterranean region. Ent. Scand., 24, 197-210. 